\title{
Optimal Operator Training Reference Models for Human-in-the-loop Systems
}

\author{
Wan-Lin Hu \\ SLAC National \\ Accelerator Laboratory, \\ Stanford University \\ wanlinhu@stanford.edu
}

\author{
Claudio Rivetta \\ Energy Sciences Directorate, \\ SLAC National \\ Accelerator Laboratory \\ rivetta@slac.stanford.edu
}

\author{
Erin MacDonald \\ Department of \\ Mechanical Engineering, \\ Stanford University \\ erinmacd@stanford.edu
}

\author{
David P. Chassin \\ Energy Sciences Directorate, \\ SLAC National \\ Accelerator Laboratory \\ dchassin@slac.stanford.edu
}

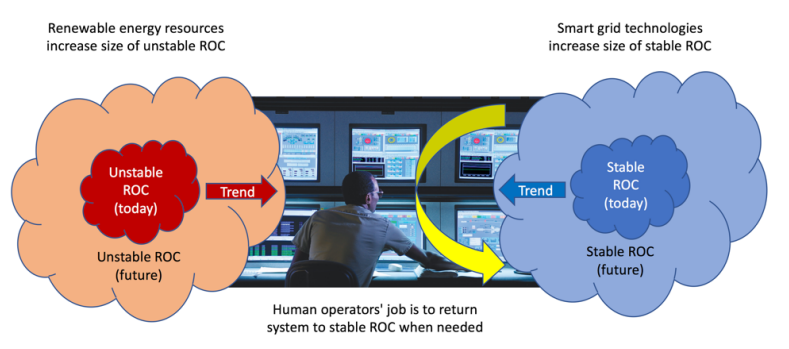

Figure 1. One role of system operators is to maintain system stability when automation cannot.

To facilitate the effectiveness of human intervention, a well-designed training and performance evaluation program is essential. Moreover, a well-defined metric of 'good performance' serving as the training goal is needed. One reasonable approach is to calculate the optimal solution for control tasks, and regard it as the performance reference. However, it is not usually possible to calculate the optimal solution for a large-scale interconnected bulk power system, which makes the idea of 'good performance' vague and ill-defined. In addition, human operator inputs are composed of both mental and physical activities. However, how human factors such as attention, learning, adaptability, and the neuromotor control of movement affect human performance also remains undiscovered in the context of power systems control. Moreover, the context-dependent characteristics of human decision making behaviors again suggest the need for a reference model of a human-in-the-loop power system. Without identifying the inherent limitations of the human operator, it is difficult to set an achievable training goal.

Efforts in human-machine systems modeling and robust control theory provide a way to address the issues discussed above. To incorporate human behaviors in a system control design, a useful practice is to model a human as a control component in the feedback control loop. This component consists of the decision making led by the mental activity and the body movement controlled by the perceptual-motor 
organization. Therefore, we can distinguish the error resulting from the decision making process and the imprecision and delay caused by neuromotor sensing and control. Despite many successes, cybernetics theory has shown that there are limitations in capturing the full breadth of human cognition and control. The theory describes human controllers as (quasi-)linear, time invariant feedback systems. Successfully applied models are those which consider human behavior in the highly-constrained compensatory tracking task [2]. However, the decision making process is context dependent, with the major literature focused on human-in-the-loop system modeling in the area of aviation and vehicle operation $[3,4,5]$. Furthermore, to address the feasibility issue of calculating the optimal solution for power system operators, and to make this optimal solution initially comparable to real human behavioral data, a simplified power operator workstation model is required.

This paper aims to describe an optimal controller with respect to a time-varying power demand for a human-in-the-loop power system experiment. This optimal controller can be regarded as the ideal human operator (i.e., a perfectly-trained operator with no adverse neuromuscular actuation effects) and serves as a training reference model for a future human-in-the-loop power system. We seek to use the optimal model with system operator training programs to reduce the likelihood and impact of operator delay and error. The ultimate goal of this research is to provide a rigorous framework for power grid control engineers to incorporate human behaviors into the control systems they must design. In this paper, we present a simple power system operator workstation that can stimulate human responses to the kind of perturbations which can occur in a power system. We present a human-in-the-loop system model with elaborated parameters, components, and signals. We develop an optimal controller for the workstation using a discrete-time $\mathcal{H}_{2}$-optimal control approach. Finally, we evaluate the performance of human subjects with respect to the optimal controller.

This paper is organized as follows. Section 2 provides background on human behaviors modeling in the controls literature, the role of human operators in power systems, and a brief review of the optimal control approach in the context of power grid. Section 3 presents the methodology to address the research objective, including workstation development, system model description, and optimal controller design. Results and discussions are presented in Section 4, followed by future work and concluding statement in Section 5.

\section{Background}

In this section, we will first review studies that model human behavior in various technical systems. We will then focus on the role of human operators in power systems. Lastly, we will propose an optimal control approach that can be used to identify a operator training reference model.

\subsection{Studies in Human Behaviors Modeling}

Extensive research has been conducted on the design of control systems with humans as the input (e.g., pilots) or the output (e.g., consumers) and achieved useful results, particularly in the areas of aviation and consumer products. Some research has been conducted on feedback systems (e.g., controllers) but with limited success in incorporating human behavior into the models of control machinery used to design and optimize systems.

In the controls literature, some research has been reported regarding systems with a human in the feedback loop (e.g., human-directed controllers). A common focus here is the decision-making of human-machine interactions. The goal is to develop an understanding of how human and machine agents can operate as teams to accomplish mission objectives $[6,7]$. However, compared with humans acting as the input and the output, research on control systems with humans in the feedback loop is less successful at incorporating human behavior since human characteristics, such as situation awareness, fatigue, panic, workload, experience level, over-confidence, and erroneous mental models can be difficult to model. On the other hand, models such as GOMS (Goals, Operators, Methods, Selection rules) [8], ACT-R [9], and Soar [10] can predict time and mental efforts but offer little for assessing errors.

\subsection{The Role of Human Operators in Power Systems}

In a modern control center, the operator monitors the grid conditions visually in real-time via the supervisory control and data acquisition (SCADA) system [11]. They take control when needed and warranted to maintain safety, security and reliability of the power system. However, human operators can also be identified as one of the contributing causes of power grid cascading failures. For example, machine-driven operator error was found to have contributed to the blackout that affected the northeastern United States and parts of Canada in 2003 [12]. Operator error was also a contributing factor to the 2-day blackout in India in 
2012 [13]. Indeed, approximately $8 \%$ of large blackouts, which affected at least 50,000 customers between 1984 and 2006, stem at least in part from operator error [14]. This strongly suggests a need to improve system-wide monitoring, alarms, and most importantly for this work, human operator training.

According to a field study conducted by Obradovich [15], the operators in the transmission control center are responsible for not only controlling voltage but also coordinating personnel, responding to emergencies, setting and assuring the power flow schedule, etc. They intervene as necessary to regulate power grids and maintain system stability when automation does not function as required or expected. However, human intervention that prevents incidents at the very early stages of the sequence of events are typically not reported [16]. This leads to a lack of understanding of how to improve the effectiveness of the human operator as a preventive safety function and how we improve the interactions between human operators and existing automation systems.

Among the various approaches to improve the human operator performance in the literature, the focus has been on enhancing the situation awareness and the human-machine interface design. Lackman and Söderlund discussed two events, Vandellos/Spain 1989 and Forsmark/Sweden 2006, in which human interventions had a significant positive effect on the event outcomes [16]. They recommended adjusting the automation to an optimum level such that operators are aware of the situation and are involved in decision making. They also recommended actively observing real-life human machine interactions to enhance our knowledge of effective human interventions. Some researchers in the field of human-computer interaction (HCI) have since focused on improving interactions between humans and computers/machines in three ways: 1) using humans working on those interfaces and analyzing the types of errors made [17]; 2) using guidelines and heuristics to evaluate user interfaces [18]; 3) using models of the human to improve the efficiency of tasks given a specific interface. HCI researchers have also developed techniques to investigate situation awareness using displays in large scale systems [19]. To apply these techniques to power systems, it is necessary to establish specific user interfaces and request the individuals to interact with these interfaces to observe and assess their joint behavior.

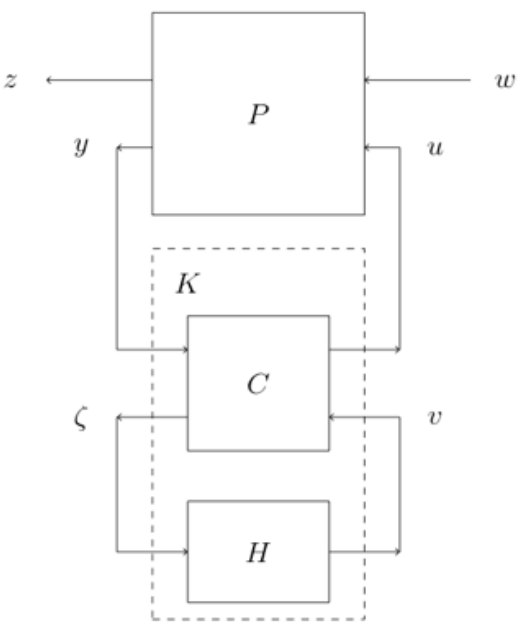

Figure 2. Standard system diagram

\subsection{Optimal Control Approach in the Context of Power Grid}

In advanced control theory, there is a useful class of control systems that include "all stabilizing parametric" controllers, such as the one shown in Figure 2. With the human operator as a feedback system element, the controller $K$ is comprised of two elements, the automatic control $C$ implemented in hardware/software and $H$ is a model of the human operators. As a whole the controller is described as $K=F(C, H)$. For such problems, the system $P$, representing the grid, is described by the state space system of equations

$$
\begin{aligned}
& \dot{x}=A x+B_{1} w+B_{2} u \\
& z=C_{1} x+D_{11} w+D_{12} u \\
& y=C_{2} x+D_{21} w+D_{22} u
\end{aligned}
$$

and the system transfer functions of $P$ in frequency domain $s=\sigma+j 2 \pi f$, are given as $\hat{p}_{i j}(s)=$ $D_{i j}+C_{i}(s I-A)^{-1} B_{j}$. It was shown [20] that if the plant $P$ satisfies certain controllability and detectability conditions, then there exists a non-empty set of all-stabilizing controllers $K$ that can be designed such that closed loop system is internally stable and the transfer function from $w$ to $z$ can be optimized. These properties for the system are achieved if the controller $H$ belongs to the space real-rational stable functions of $s$.

This observation is significant: as long as we can describe the controller $H$ using a similar mathematical construct as the plant $P$ and the control system $C$, then we are assured that every possible parametric realization of $H$ will give rise to a stable system response to every possible system disturbance. 
If we consider $P$ to be a linear time-invariant (LTI) model of bulk electric power interconnection, $C$ is an LTI model of its control system, and $H$ is an LTI model of the human operators, then we can show that it is possible to restrict the behavior of the human operators that could give rise to an unstable system response following a disturbance.

A second important result is that if the output $z$ is designed as a cost function and the closed-loop system connecting $C$ and $P$ satisfies certain conditions, then there exists a unique controller $H_{o p t}$ that will minimize the magnitude of $z$ according to some particular norm, e.g., $\mathcal{H}_{2}, \mathcal{H}_{\infty}$, for a given input disturbance $w$. The optimal controller $\mathcal{H}_{\text {opt }}$ can be regarded as the ideal human operator and its performance can be regarded as that of a perfectly-trained human operator.

\section{Methodology}

In this paper we examine a simple prototype of a HCI design, and how human subjects compare to an optimal model of the system. The simple system is not intended to accurately represent a power system. Rather it is intended to facilitate our understanding of how effective the proposed strategy of comparing human operators to a theoretical optimal operator will be in formulating a training regimen for system operators in general.

We propose to jointly describe an idealized power system and its control system using the following procedure: (1) develop a simple power system operator workstation using a real-time simulator which supports system identification on the operator performing simple tasks; (2) design an all-stabilizing controller $K$ for the workstation $\mathrm{HCI}$ and place it "in the loop" of the simulator; (3) evaluate the performance of optimal controller, which can be regarded as the ideal operator.

\subsection{Simulated Operator Workstation Development}

To design an optimal controller that can be used to train operators in the power system context, we first established a simple computer-based operator workstation (i.e., simulated power dispatch system), which interacts with human subjects and thus allows us to analyze human control behaviors responding to system disturbances. Human operators will serve as control elements in the simulated system. Their task will be to control the power generator output power to maintain the system frequency at $60 \mathrm{~Hz}$, with the time-varying system load as the stimulus and the current system frequency as the feedback signal (Fig. 3). The stimulus was selected to be rich enough for system identification yet steady enough for humans to learn

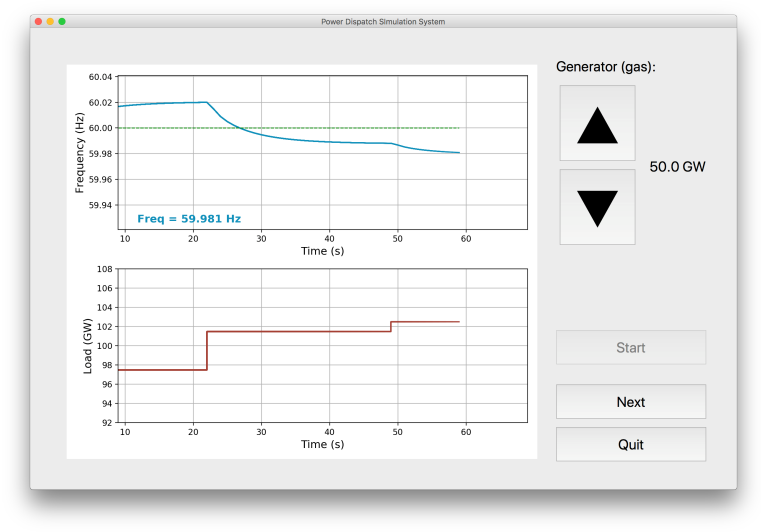

Figure 3. The screenshot of the simulation control task

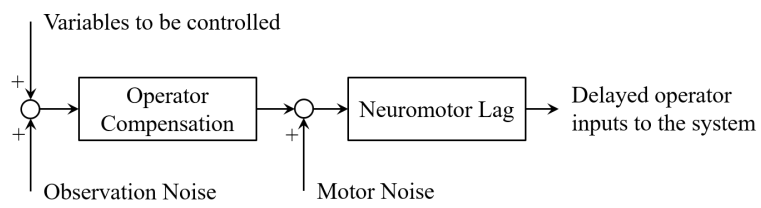

Figure 4. Block diagram of a human operator model for the developed workstation

quickly.

The operator control action for this system can be depicted by the block diagram shown in Fig. 4. The input is the observation of the signal that the operator is trying to control, in conjunction with an observation noise since the operator cannot observe signals with unlimited accuracy. Based on the observation, the operator attempts to maximize the system performance (i.e., the operator compensation on the diagram), which includes the decision-making led by the mental activity and the physical control action (e.g., input device manipulation to adjust the generator output). Similarly, the operator is incapable of manipulating the input device with unlimited accuracy, thus a motor noise is introduced at this stage, which is added to a delay in the operator inputs to the system. The neuromotor lag is due to the band-limited nature of the operator's neuromuscular system [3]; hence, it does not differ much between simple and complex control tasks. This also holds true for the observation and motor noise. This characteristic suggests that the input delay modeled for the simple workstation would still be valid in complex scenarios.

The optimal control response developed in this paper will serve as the training reference model to characterize the human control led by the mental activity. The optimal solution itself may vary with the complexity 
or factors of a task, but the methodology to obtain the optimal operator training reference model is expected to be generalized.

\subsection{System Model}

The control system design is determined using the following procedure:

1. A vector of observable states $x$ of the system is identified.

2. The optimal performance objective is identified as a $\mathcal{H}_{2}$-norm ${ }^{1}$ of a vector $z$, which can be derived from the observable states $x$ of the system.

3. A vector $u$ of inputs from the controller (i.e., the human control signal) is identified.

4. An input disturbance $w$ is specified.

5. An all-stabilizing controller $C$ defined by the gains $F$ and $L$ such that $A+B_{2} F$ and $A+L C_{2}$ are stable and

$$
\begin{aligned}
\dot{x}_{c} & =\left(A+B_{2} F+L C_{2}\right) x_{c}-L y-B_{2} \nu \\
u & =F x_{c}-\nu \\
\zeta & =-C_{2} x_{c}+y
\end{aligned}
$$

A parametric controller $H$, representing the human operator behavior, is designed so that the transfer matrix from $w$ to $z$ is given as $\hat{t}_{11}(s)+\hat{t}_{12}(s) H(s) \hat{t}_{21}(s)$, where $\hat{t}_{11}, \hat{t}_{12}$ and $\hat{t}_{21}$ are the closed-loop transfer functions of the system $T=(C, P)$ taken from $(w, \nu) \rightarrow(z, \zeta)$.

The $\mathcal{H}_{2}$-optimal control design problem is solved for combined system $K_{o p t}=\left(C, H_{o p t}\right)$ given the plant $P$, from which the transfer function for $H_{o p t}$ is identified. The parameters of the optimal operator are mapped to characteristics corresponding to the principal response components of human performance, i.e., delays, errors, biases, magnitudes, etc. These can be assessed through monitoring and either modified through a training regimen.

Therefore, a human-in-the-loop power system model can be illustrated as Fig. 5. The components included in the system are the optimal (human) operator $H$ worked together with the automatic control $C$, the disturbance response $D$, the generator response $P$, the system response $R$, the measurement noise $K_{n}$, and the frequency deviation cost $K_{z}$.

\footnotetext{
${ }^{1}$ Although we have chosen to use the $\mathcal{H}_{2}$-norm in this paper, the reader will note that the methodology is similar if implemented using an $\mathcal{H}_{\infty}$-norm.
}

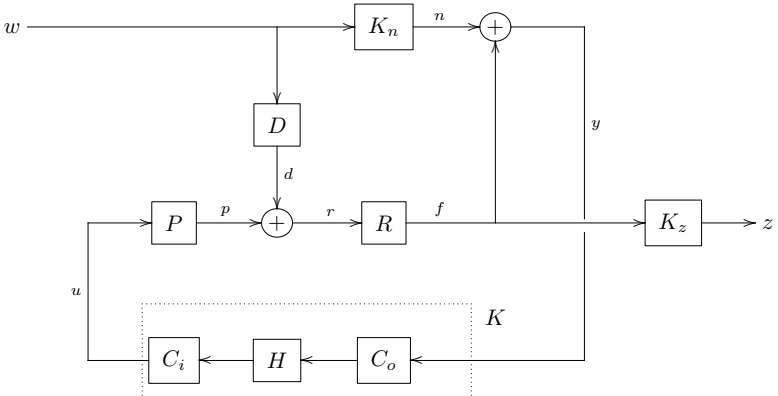

Figure 5. Human-in-the-loop system diagram for a

\begin{tabular}{|c|c|c|}
\hline Name & Value & Description \\
\hline$a$ & $=1.0 \mathrm{sec}$ & Generator time constant \\
\hline$b$ & $=10.0 \mathrm{sec}$ & System time constant \\
\hline$c$ & $=50.0 \mathrm{sec}$ & Disturbance time constant \\
\hline$k_{n}$ & $=0.01$ & Measurement noise magnitude \\
\hline$k_{z}$ & $=5.0$ & $\begin{array}{l}\text { Frequency cost magnitude (p.u. } \\
\text { input cost) }\end{array}$ \\
\hline
\end{tabular}
model power system.

Table 1. System diagram parameters

\subsection{Optimal Controller Design}

Based on the system model, we describe the state-space approach to a discrete-time $\mathcal{H}_{2}$-optimal control problem for the system shown in Figure 2 according to [21]. The input $w$ is standard white-noise. The challange is to design a controller $K$ that stabilizes $P$ and minimizes the root-mean-square value of $z$, the $\mathcal{H}_{2}(\mathbb{D})$-norm of the transfer matrix from $w$ to $z$ [22].

The continuous-time state-space model for the parameters given in Table 1 , is given in the standard form

$$
\left[\begin{array}{c}
\dot{x}_{1} \\
\dot{x}_{2} \\
\dot{x}_{3} \\
z \\
u \\
y
\end{array}\right]=\left[\begin{array}{ccc|cc}
-1 & 0 & 0 & 0 & 1 \\
1 & -0.1 & -0.16 & 0 & 0 \\
0 & 0 & -0.02 & 0.12 & 0 \\
\hline 0 & 5 & 0 & 0 & 0 \\
0 & 0 & 0 & 0 & 1 \\
0 & 1 & 0 & 0.01 & 0
\end{array}\right]\left[\begin{array}{c}
x_{1} \\
x_{2} \\
x_{3} \\
w \\
u
\end{array}\right]
$$

and the optimal operator response transfer function is

$$
K(s)=\frac{\hat{u}(s)}{\hat{y}(s)}=\frac{-18.8(s+0.528)(s+1)}{\left(s^{2}+6.03 s+15.6\right)(s+0.023)}
$$

\section{Results and Discussion}

We verify the performance of the designed controller under two conditions: 1) given an impulse 


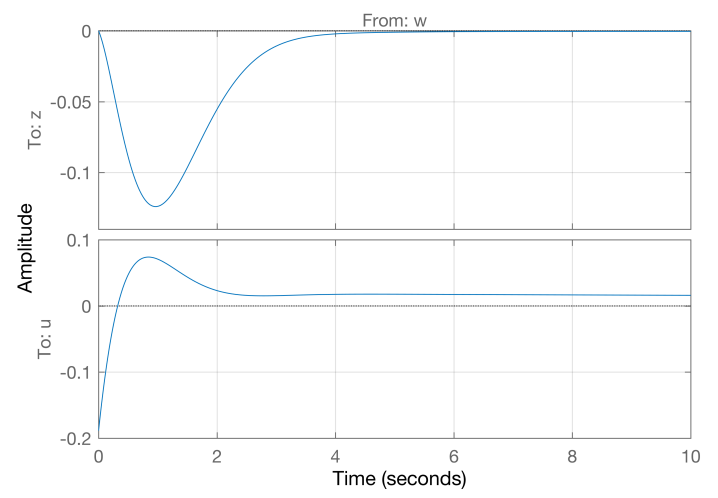

Figure 6. Response of the optimal operator to a system event.

representing the outage event, and 2) given a 600-second time-varying load representing an usual operation condition. This load sequence is used in the workstation introduced in Section 3.1.

The impulse response for the complete system is shown in Fig. 6. A disturbance $w$ (an impulse input) is applied to the system, and the controller is capable of stabilizing the system and minimizing the root-mean-square value of the frequency response cost $z$. Note that the disturbance transfer function $D(s)$ is used to provide the power deviation, including the response from the rest of the bulk power system.

Although human operators are not expected to provide this kind of fast response, the model system provides a very useful baseline with which to study human responses and the susceptibility of operators to a training regimen with a well-known optimal response objective.

We computed the optimal response to a time-varying system load, and then compared the optimal response to the responses of two naive human subjects. The load variation, generator output power (signal $p$ in Fig. 5), and the corresponding frequency deviation are shown in Fig. 7. The costs of these response are listed in Tab. 2. The optimal response was smooth and fast, resulting in a cost that was approximately ten times less than that of humans. However, there are observation noise, motor noise, and neuromotor lag on a human control action as illustrated in Fig. 4. Therefore, this optimal response is an ideal case of only the mental decision making process. Nonetheless, there are some interesting findings from the example human responses. Subject 1 adjusted the system input fast and frequently. On the other hand, subject 2 adjusted the system input more smoothly. By observing the corresponding frequency deviation, a fast input with a tolerance of overshoot in

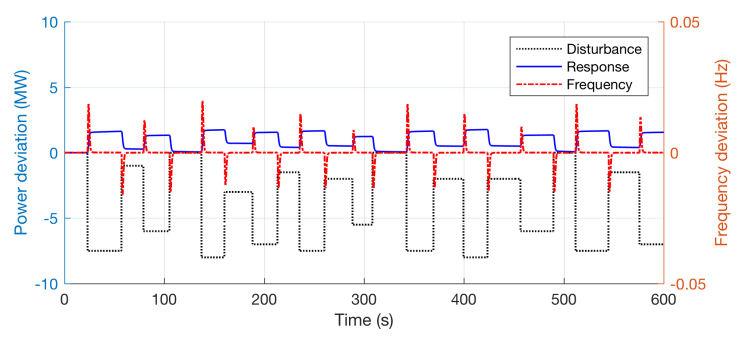

(a) Optimal response

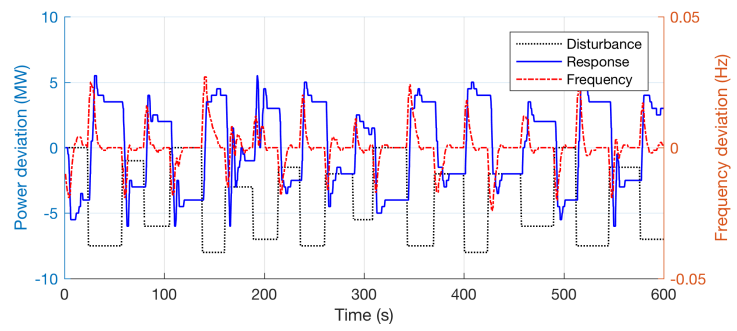

(b) Example human response 1

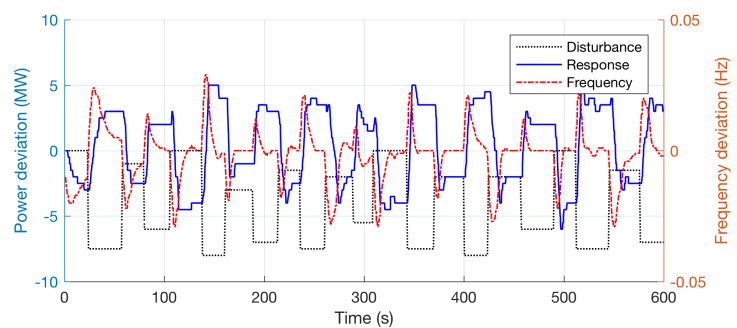

(c) Example human response 2

Figure 7. Example of (a) the optimal response and (b) (c) human responses to a time-varying system load. The black line is the disturbance (i.e., the load demand). The blue line is the generator output (i.e., signal $p$ in Fig. 5). The red line is the resulting

frequency deviation. The lower the deviation, the better the controller performance.

the output (i.e., frequency deviation) would make the system performance more close to optimal.

In this given task, human operators anticipate system outputs and then compare this expectation with the observation (i.e., the actual outputs). They then adjust their control inputs based on their trained response. Operators must be aware of the dynamically changing state of the system load and frequency, and project the perceived status of these elements in the near future. This process involves domain knowledge [23] and situation awareness [24]. Moreover, mental workload [25] and expertise [26] also could affect the performance. Individuals who know how the system works, have experience in operation, are more alert, or concentrate more on the situation are more likely 
Table 2. The frequency costs of the human subject responses and the optimal response

\begin{tabular}{cc}
\hline \hline Operator & Cost \\
\hline Optimal & 0.017 \\
Human subject 1 & 0.145 \\
Human subject 2 & 0.269 \\
\hline \hline
\end{tabular}

to achieve good performance. Therefore, we expect to see individual differences in this power operation task. Indeed, we observed differences between our two human subjects as discussed above. This highlights a need to incorporate these factors into the optimal reference model, or to customize the training program. For example, setting sequential goals for individuals based on the years of their domain experience or on their shift schedule may help us obtain more 'realistic' optimal reference models. We anticipate that the wider domain experience can be engaged in various power control tasks and not just system frequency regulation. Instead of simply responding to events, the role of operators in a modern power transmission network is evolving more towards management and supervision. Operators increasingly forecast and plan to mitigate potential problems (e.g., ramp capacity adjustment), and their experience is assumed to affect their performance.

Furthermore, in this simplified task, human operators extract information from a single source of visual information. However, in a real-life control room, operators need to process various information from different resources with limited attention capacity. Processing multiple or simultaneous stimuli is not a challenge for an optimal controller, but it could impact the human's performance. Therefore, one direction to enhance the effectiveness of the optimal reference model is to take the amount and exposure time of information into account.

\section{Conclusion and Future Work}

This research seeks to enhance the power operator training programs by proposing a methodology to design the optimal performance reference model. This optimal controller can be regarded as the ideal operator and serves as a training metric for human-in-the-loop power systems. To make the optimal model comparable with human behavioral data, we have developed a simple computer-based operator workstation, in which a time-varying system load is the stimulus. The control goal in this simplified system is to minimize the frequency deviation. We presented an overall model for the human-in-the-loop power system, and applied the optimal controller synthesis methodology to the model system to design the optimal controller. Results suggest that this optimal controller performed well in response to both the impulse disturbance and a period of time-varying load condition.

The current system design assumes that there are no delays in the human-computer interface. Future work will involve adding a discrete-time user interface and input/output delays. Next steps will also include establishing a mathematical model to describe the human control behaviors, so the training progress can be assessed quantitatively.

This capability is intended to support the development and integration of solar, storage and demand response technologies. These energy technologies present operators with more challenging contingencies and perhaps also more effective mitigation strategies. These challenges and strategies will inevitably become a part of the operator "playbook" and this work is intended to give utilities and control area training programs a technical foundation based on both robust control theory and human-computer systems engineering, all of which supports grid modernization efforts at the regional and national level.

\section{Acknowledgments}

SLAC National Accelerator Laboratory is operated for the US Department of Energy by Stanford University under Contract No. DE-AC02-76SF00515. The authors would also like to thank Tom Sandquist and Jean Sholtz of Pacific Northwest National Laboratory for their insights and contributions to the genesis of this work.

\section{References}

[1] D. J. Hill and G. Chen, "Power systems as dynamic networks," 2006 IEEE International Symposium on Circuits and Systems, pp. 722-725, 2006.

[2] M. Mulder, D. Pool, D. Abbink, E. Boer, E. Boer, P. Zaal, F. Drop, K. van der El, and R. van Paassen, "Manual control cybernetics: State-of-the-art and current trends," IEEE Transactions on Human-Machine Systems, vol. DOI: 10.1109/THMS.2017.2761342, 2017.

[3] D. B. Doman and M. R. Anderson, "A fixed-order optimal control model of human operator response," Automatica, vol. 36, pp. 409-418, 2000.

[4] N. Kuge, T. Yamamura, O. Shimoyama, and A. Liu, "A Driver Behavior Recognition Method Based on a Driver Model Framework," tech. rep., 1998.

[5] S. Sharma, H. Singh, and A. Prakash, "Multi-agent modeling and simulation of human behavior in aircraft evacuations," IEEE Transactions on Aerospace and Electronic Systems, vol. 44, no. 4, pp. 1477-1488, 2008.

[6] J. Baillieul, N. E. Leonard, and K. A. Morgansen, "Interaction dynamics: The interface of humans and smart machines," Proceedings of the IEEE, vol. 100, no. 3, pp. 567-570, 2012. 
[7] R. Arkin, Governing lethal behavior in autonomous robots. CRC Press, 2009.

[8] D. Diaper and N. Stanton, The handbook of task analysis for human-computer interaction. CRC Press, 2003.

[9] J. R. Anderson, B. E. John, M. A. Just, P. A. Carpenter, D. E. Kieras, and D. E. Meyer, "Production system models of complex cognition," in Proceedings of the seventeenth annual conference of the cognitive science society, vol. 17, p. 9, Psychology Press, 1995.

[10] M. A. Cohen, F. E. Ritter, and S. R. Haynes, "Herbal: A high-level language and development environment for developing cognitive models in soar," in Proceedings of the 14th Conference on Behavior Representation in Modeling and Simulation, vol. 1334140, 2005.

[11] J. Giri, M. Parashar, J. Trehern, and V. Madani, "The situation room: Control center analytics for enhanced situational awareness," IEEE Power and Energy Magazine, pp. 24-39, 2012.

[12] B. S. H. Horowitz, A. G. Phadke, B. A. Renz, S. Horowitz, A. G. Phadke, and B. A. Renz, "The Future of Power Transmission," IEEE Power and Energy Magazine, pp. 34-40, 2010.

[13] C. D. Brummitt, P. D. H. Hines, I. Dobson, C. Moore, and R. M. D'Souza, "Transdisciplinary electric power grid science," Proceedings of the National Academy of Sciences, vol. 110, no. 30, p. 12159, 2013.

[14] P. Hines, K. Balasubramaniam, and E. C. Sanchez, "Cascading failures in power grids," IEEE Potentials, pp. 24-30, 2009

[15] J. H. Obradovich, "Understanding cognitive and collaborative work: Observations in an electric transmission operations control center," in Proceedings of the Human Factors and Ergonomics Society 55th Annual Meeting, pp. 247-251, 2011.

[16] T. O. Lackman and K. Söderlund, "Situations saved by the human operator when automation failed," Chemical Engineering Transactions, vol. 31, pp. 385-390, 2013.

[17] J. S. Dumas and J. Redish, A practical guide to usability testing. Intellect books, 1999.

[18] J. Nielsen and R. Molich, "Heuristic evaluation of user interfaces," in Proceedings of the SIGCHI conference on Human factors in computing systems, pp. 249-256, ACM, 1990

[19] M. R. Endsley, "Design and evaluation for situation awareness enhancement," in Proceedings of the Human Factors Society annual meeting, vol. 32.2, pp. 97-101, SAGE Publications Sage CA: Los Angeles, CA, 1988.

[20] T. Chen and B. Francis, "Optimal control of sampled-data systems," 1995.

[21] J. C. Doyle, K. Glover, P. P. Khargonekar, and B. A. Francis, "State-space solutions to standard h2 and h infin; control problems," IEEE Transactions on Automatic Control, vol. 34, pp. 831-847, Aug 1989.

[22] K. Zhou, J. C. Doyle, K. Glover, et al., Robust and optimal control, vol. 40. Prentice hall New Jersey, 1996.

[23] D. N. Hogg, K. Follesø, F. Strand-Volden, and B. Torralba, "Development of a situation awareness measure to evaluate advanced alarm systems in nuclear power plant control rooms," Ergonomics, vol. 38, no. 11, pp. 2394-2413, 1995.
[24] M. R. Endsley and C. A. Bolstad, "Individual Differences in Pilot Situation Awareness," The International Journal of Aviation Psychology, vol. 4, pp. 241-264, jul 1994.

[25] S.-L. Hwang, Y.-J. Yau, Y.-T. Lin, J.-H. Chen, T.-H. Huang, T.-C. Yenn, and C.-C. Hsu, "Predicting work performance in nuclear power plants," Safety Science, vol. 46, no. 7, pp. 1115-1124, 2008.

[26] F.-H. Huang, Y.-L. Lee, S.-L. Hwang, T.-C. Yenn, Y.-C. Yu, C.-C. Hsu, and H.-W. Huang, "Experimental evaluation of human-system interaction on alarm design," Nuclear Engineering and Design, vol. 237, no. 3, pp. 308-315, 2007. 